\title{
Affiliative Behavior Requires Juvenile, But Not Adult Neurogenesis
}

\author{
Lan Wei, ${ }^{1}$ Michael J. Meaney, ${ }^{2,3}$ Ronald S. Duman, ${ }^{1}$ and Arie Kaffman ${ }^{1}$ \\ ${ }^{1}$ Department of Psychiatry, Yale University School of Medicine, New Haven, Connecticut 06511, ${ }^{2}$ Sackler Program for Epigenetics and Psychobiology at \\ McGill University, Douglas Mental Health University Institute, Montreal, Quebec, H4H 1R3 Canada, and ${ }^{3}$ Singapore Institute for Clinical Sciences, \\ Singapore 117609
}

The capacity to interact with conspecifics is essential for stable social networks, reproduction, and survival in mammals. In rodents, social exploration and play behavior increase during the juvenile period, suggesting that this timeframe represents an important window for socialization. However, the cellular and molecular mechanisms necessary to support this developmental process have not been elucidated. Neurogenesis during the juvenile period, like that in adults, is mainly confined to the subgranular and subventricular zones. Nevertheless, the levels of neurogenesis are significantly higher during the juvenile period, suggesting unique functions not shared with adult neurogenesis. Here we use a transgenic mouse approach that allows for ablation of neurogenesis during different developmental phases. We find that ablating neurogenesis during either juvenile or adult phases altered anxiety and memory in adult female mice, demonstrating an age-independent function of new neurons for certain behaviors. Blocking neurogenesis during the juvenile period resulted in a profound impairment in the ability of these mice to interact with other adult females or to retrieve pups, without causing gross olfactory deficits. Interestingly, ablating neurogenesis in adult females had no effect on these social behaviors. This work defines a novel role for juvenile neurogenesis in establishing brain circuits necessary for socialization, and demonstrates that juvenile and adult neurogenesis make different contributions to social competency in adult female mice. Additional work is needed to determine whether ablation of juvenile neurogenesis in the subgranular zone and/or the subventricular zone is responsible for the social abnormalities seen after global elimination of juvenile neurogenesis.

\section{Introduction}

Neurogenesis in the adult brain is mainly confined to the dentate gyrus (DG) of the hippocampus and the olfactory bulb (Doetsch and Hen, 2005). Immature neurons generated in the subgranular zone (SGZ) differentiate into granule cells that integrate into the DG of the hippocampus and are required for some hippocampaldependent tasks (Li et al., 2009). Neural stem cells (NSC) generated in the subventricular zone (SVZ) migrate and differentiate along the rostral migratory stream, giving rise to new interneurons in the main olfactory bulb (MOB). Most of these inhibitory neurons are generated during the first 2 weeks of life, but significant production continues in mammals throughout the juvenile period (i.e., the third through eighth weeks of life) and in adulthood (Lemasson et al., 2005; Batista-Brito et al., 2008). To date, no deficits in olfactory discrimination tasks have been identified after ablating MOB neurogenesis in adulthood (Lazarini et al.,

\footnotetext{
Received March 16, 2011; revised Aug. 12, 2011; accepted Aug. 16, 2011.

Author contributions: A.K. designed research; L.W. and A.K. performed research; L.W. and A.K. analyzed data; M.J.M., R.S.D., and A.K. wrote the paper.

This work was supported by NIMH Grant 1K08MH074856, NARSAD Young Investigator Award 2004, APIRE/ Wyeth M.D.;Ph.D. Psychiatric Research Award 2005, and the APIRE/Merck Early Academic Career Research Award 2006. We thank M. Picciotto, D. Guarnieri, and R. DiLeone for helpful comments on the manuscript.

The authors declare no financial conflicts of interest.

Correspondence should be addressed to Arie Kaffman, 300 George Street, Suite 901, New Haven, CT 0651. E-mail: arie.kaffman@yale.edu.

DOI:10.1523/JNEUROSCI.1333-11.2011

Copyright $\odot 2011$ the authors $\quad 0270-6474 / 11 / 3114335-11 \$ 15.00 / 0$
}

2009; Valley et al., 2009). However, deficits in short- (BretonProvencher et al., 2009) and long-term (Lazarini et al., 2009) olfactory memories, as well as impairment in conditioned fear response (Valley et al., 2009), have been reported after adult ablation of $\mathrm{MOB}$ neurogenesis, suggesting a role in processing certain olfactory information.

Affiliative behavior is associated with the induction of adult neurogenesis in rodents. For example, social cues present in the urine of a dominant male increase neurogenesis in both the DG and the MOB in female mice and determine preference to associate with a dominant male (Mak et al., 2007). Neurogenesis in the MOB increases during the postpartum period, suggesting a role in maternal behavior (Shingo et al., 2003). Finally, mating increases neurogenesis in the monogamous prairie vole female, demonstrating another association with social attachment (Fowler et al., 2002).

The major developmental processes that characterize postnatal life, including myelogenesis (Mathis et al., 2000), DG formation, and cerebellar neurogenesis, are completed approximately at postnatal day (PND) 30, confining neurogenesis in the juvenile brain, like the adult, to the SVZ and SGZ (Lemasson et al., 2005; Li et al., 2009). Nevertheless, very little is known about the role that juvenile neurogenesis plays in guiding the development of complex behaviors in adulthood. To address this question, we generated transgenic mice that expressed the thymidine kinase (TK) gene fused to the GFP (TK-GFP) in NSC. TK phosphorylates the nucleoside analog Ganciclovir (GCV), which is then 
incorporated into newly synthesized DNA, leading to chain termination and apoptosis in mitotically active cells (Bush et al., 1998). Administrating a low dose of GCV directly into the lateral ventricle allowed for rapid and complete elimination of neurogenesis without the toxicity associated with peripheral administration of GCV. Female mice were used because of our interest in assessing pup retrieval and because of studies suggesting that female-female social interactions play an important role in regulating the menstrual cycle and maintaining stable social hierarchy within a colony (Drickamer, 1974; Maggio and Whitney, 1985). Here we show that juvenile neurogenesis is required to support the normal increase in socialization seen during the juvenile period, but once social behavior has matured in adulthood, it no longer requires adult neurogenesis.

\section{Materials and Methods}

Generating TK-GFP transgenic mice. A $663 \mathrm{bp}$ fragment containing the second intron enhancer of the nestin gene was used to drive expression of the TK-GFP. The entire $2.9 \mathrm{~kb}$ fragment containing the nestin enhancer, the TK gene fused in frame to the GFP, and the SV40 poly A tail was sequenced, purified, and used for pronuclei injections. Of the 40 founders generated, four founders carried the transgene. The expression pattern of TK-GFP in all four lines was similar except that line 43 had no expression of GFP outside the SGZ, SVZ-MOB regions. Line 43 was then backcrossed to BALB/cByj (The Jackson Laboratory) for at least 12 generations to generate an isogenic line of mice on this background that was used in these experiments.

Animals. Mice where maintained under a 12:12 h light:dark cycle with lights on at 7:00 $\mathrm{AM}$ and a constant temperature of $22^{\circ} \mathrm{C}$ with $50 \%$ humidity. All the work reported here was done in gonad-intact females and no attempt was made to synchronize the estrus cycle. Studies were approved by the Institutional Animal Care and Use Committee at Yale University and were conducted in accordance with the recommendations of the NIH Guide for the Care and the Use of Laboratory Mice.

Surgeries. For subcutaneous administration of GCV, osmotic minipumps $(0.25 \mu \mathrm{l} / \mathrm{h}$; catalog no. 2004; Alzet) were filled with $500 \mathrm{mg} / \mathrm{ml}$ GCV (Cytovene; Roche) dissolved in sterile PBS and primed according to the manufacturer's instructions. TK-GFP or wild-type mice were briefly anesthetized with $2 \%$ isoflurane delivered at $2 \mathrm{~L} / \mathrm{min}$ oxygen flow. A small incision was made along the animal's back and the pump was placed in a subcutaneous pocket. The skin was then sutured and the animal was weighed and allowed to recover. For intracerebroventricular surgeries, osmotic minipumps $(0.11 \mu \mathrm{l} / \mathrm{h}$; catalog no. 1004; Alzet) were filled with $100 \mathrm{mg} / \mathrm{ml} \mathrm{GCV}$ dissolved in sterile PBS, connected to a cannula (brain kit no. 3; Alzet) via $3 \mathrm{~cm}$ tubing, and primed according to the manufacturer's instructions. Mice were anesthetized with $2 \%$ isoflurane $/ 2 \mathrm{~L} / \mathrm{min}$ oxygen and placed in a stereotaxic frame equipped with an isoflurane nose cone (Stoelting). A skin incision was made to expose the skull and a small pocket for inserting the pump was made along the back midline. A $1 \mathrm{~mm}$ hole for cannula placement was drilled using the following coordinates from bregma: lateral medial axis, $1.1 \mathrm{~mm}$; anteroposterior axis, $0 \mathrm{~mm}$. An additional hole was drilled at the occipital bone for placing a security screw. The minipump was then placed in the subcutaneous pocket and the skull was dried with acetone followed by application of superglue. Next, the cannula was lowered to a depth of $3 \mathrm{~mm}$ and then slowly raised to a final $z$-coordinate of $2.5 \mathrm{~mm}$ from the top of the skull. A small security screw was placed and the entire surface was covered with Gerystore dental cement (Den-Mat). The skin was then sutured and the animal was weighed and allowed to recover.

Subcutaneous versus intracerebroventricular administration of GCV. To assess the relative toxicity of subcutaneous versus intracerebroventricular administration of Ganciclovir, adult TK-GFP and wild-type littermates ( $n=5$ for each genotype for intracerebroventricular treatment and $n=4$ for each genotype for subcutaneous treatment) were exposed for $30 \mathrm{~d}$ to either $3 \mathrm{mg}$ of $\mathrm{GCV}$ per day delivered subcutaneously (i.e., 100 $\mathrm{mg} / \mathrm{kg}$ per day), or $0.26 \mathrm{mg}$ of GCV per day delivered intracerebroventricularly. To calculate weight gain over the course of treatment, mice were weighed before surgery and after $30 \mathrm{~d}$ of exposure to GCV (i.e., weight posttreatment - weight before GCV treatment).

Juvenile and adult ablation of neurogenesis. For experiments involving juvenile ablation of neurogenesis, mice ( $n=7$ for TK-GFP and $n=7$ for wild-type littermates) were weaned on PND 22 and housed three or four mice per cage. At PND 27-35, these mice underwent intracerebroventricular surgeries, after which they were singly housed for $30 \mathrm{~d}$ until behavioral testing began. Single housing the mice was necessary to maintain GCV infusion as cage mates chewed on any exposed tubing. For experiments involving adult ablation of neurogenesis, a total of 13 mice were used ( $n=6$ for TK-GFP and $n=7$ for wild-type littermates). These were housed in groups of three or four mice per cage until PND 90-120, at which point they underwent intracerebroventricular surgeries and were then singly housed for $30 \mathrm{~d}$ until behavioral testing began.

Social development studies. To characterize social behavior during the juvenile period, a group of PND $21 \mathrm{BALB} / \mathrm{cByj}$ females $(n=22)$ were purchased from The Jackson Laboratory. These mice were weaned on PND 21 and delivered without their dams to our facility where they were group housed until PND 27. On PND 27, all mice were singly housed until tested in the social affiliation assay, latency to find food, and exploration of inanimate object on PND $35(n=7)$, PND $55(n=8)$, or PND $75(n=7)$. In addition, a group of 15 TK-GFP underwent intracerebroventricular surgeries on PND 27 and were exposed to either $260 \mu \mathrm{g} / \mathrm{d}$ GCV in PBS $(n=8)$ or PBS alone $(n=7)$, singly housed, and tested on PND 75 in the social affiliation assay, latency to find food, and exploration of inanimate object.

Behavioral studies. Behavioral experiments were performed under dim lighting conditions (15-30 Lux in the arena) to reduce anxiety and light sensitivity. Behavioral testing was conducted daily, between 1:00 and 5:00 P.M., in this order: open field, dark-light, object recognition, affiliative behavior, pup retrieval, latency to find food, and odor discrimination.

Open-field exploration. Open-field exploration was done as described previously (Wei et al., 2010). In brief, individual female mice were allowed to freely explore a black $50 \times 50 \mathrm{~cm}$ Plexiglas arena for $5 \mathrm{~min}$. Distance traveled and the amount of time the mouse spent in the inner 15 $\mathrm{cm}$ area were determined using the EthoVision tracking system (Noldus Information Technology).

Dark-light choice. Dark-light choice was done as described previously (Henderson et al., 2004). In brief, a $44 \times 20 \times 20 \mathrm{~cm}$ Plexiglas cage was partitioned so that two-thirds of the length were white-walled and illuminated with a light source at 15 Lux while one third had black walls and was completely darkened (1 Lux) except for a small opening connecting the two compartments. Mice were initially placed in the white compartment and allowed to explore the box freely for 10 min while being videotaped. An observer who was unaware of the genotype of the mouse scored the total number of transitions between the two compartments and time spent in the light compartment.

Object recognition. Mice were habituated to an opaque chamber $(18 \times$ 9.5 inch) where two identical objects were placed at opposite sides of the cage for $15 \mathrm{~min}$ on two consecutive days. On the third day, the mouse was returned to the same chamber except that one of the objects was replaced with a novel object. The session was videotaped for $5 \mathrm{~min}$. The position and the identity of the novel object were randomized to eliminate object and position biases. The amount of time the mouse explored each object was scored by a rater who was blind to the mouse's genotype; the score was used to calculate percentage preference index. The percentage preference index $(\mathrm{PI})$ was calculated as follow: $\% \mathrm{PI}=$ (time exploring novel object $\times 100) /$ time exploring both objects.

Social affiliation test. The subject mouse (i.e., WT or TK-GFP) was allowed to explore an $18 \times 9.5$ inch cage layered with bedding for $15 \mathrm{~min}$ followed by the addition of an unfamiliar, 8- to 10-week-old BALB/cByj female object mouse for $5 \mathrm{~min}$. A small black mark was painted on the back of the object mouse to help distinguish it from the subject mouse. The session was then videotaped and the following behaviors were scored during the first $3 \mathrm{~min}$ of the session by an observer who was blind to the mice's genotype: time the subject mouse actively explored the object mouse (i.e., sniffing, following, pushing under), time in close proximity (defined as the time that the subject and object mice were $<2 \mathrm{~cm}$ apart), 
and number of times the subject animal evaded attempts by the object mouse to engage in social contact.

Pup retrieval. Three BALBc/cByj pups (PND 1-3) were scattered in the home cage of a nulliparous female and the session was videotaped for 15 min and scored by a rater who was unaware of the animal's genotype. Failure to retrieve pups was defined as the inability to transfer even one pup into the nest within the allotted $15 \mathrm{~min}$ of the session and the absence of maternal behavior toward the pups during the session (i.e., huddling over or licking and grooming), while retrieval behavior was assigned to mice that transferred at least one pup into their nests and huddled over and cared for the pups during the 15 min session.

Latency to find food. Before testing, the mice were transferred to a new cage containing four cups of fresh corncob bedding, providing a $2 \mathrm{~cm}$ layer of bedding in the home cage. The chow was then removed but water was available ad libitum. At the end of the $18 \mathrm{~h}$ food-deprivation period, the mouse was transferred into a holding cage and a small $(1.0 \mathrm{~g})$ chow pellet was buried under the bedding in its home cage. The mouse was then returned to its home cage and the latency to locate and start eating the food was recorded.

Odor-discrimination task. Two q-tips were attached to two opposing walls of a $16 \times 9$ inch arena so they extended horizontally 2 inches above the arena's floor. One hundred microliters of either female urine or water were applied to each q-tip and the location of each odorant was randomized to minimize position bias. Mice were individually placed in the middle of the arena and the session was recorded for $5 \mathrm{~min}$. The time the mouse sniffed each q-tip during the first $2 \mathrm{~min}$ of the session was scored and the PI for sniffing urine was calculated as (time sniffing urine $\times$ 100)/total amount sniffing the two q-tips. Note that female mice with intact sense of smell will preferentially smell urine (i.e., PI $>50 \%$ ).

Inanimate toy exploration. Mice were placed individually in an $18 \times 9.5$ inch arena. After $15 \mathrm{~min}$ of acclimation to their new environment, an inanimate rubber toy ( $\sim 5 \mathrm{~cm}$ in size $)$ was added to the arena. The session was videotaped for $5 \mathrm{~min}$ and the time the animal explored the object during the first $3 \mathrm{~min}$ of the session was scored. This was a similar procedure as the one described for the social affiliation test, except that a toy was added to the arena instead of an unfamiliar conspecific.

Affiliative behavior and the estrus cycle. To test the effect of the estrus cycle on affiliative behavior, a group of 10-week-old BALB/cByj females $(n=27)$ were purchased from The Jackson Laboratory and housed three to five mice per cage upon arrival. After 1 week of acclimation, the mice were singly housed for $6 \mathrm{~d}$ and tested in the social affiliation test. At the completion of the behavioral testing, the menstrual cycle of the mouse (e.g., proestrus, estrus, metestrus, diestrus) was determined using vaginal smear as described previously (Caligioni, 2009). We then selected the five mice that showed the highest levels of social exploration on day 1 (i.e., high-social score group) and the five mice that showed the lowest amount of time investigating an unfamiliar conspecific on day 1 (i.e., low-social score group) and repeated the same procedure for 4 consecutive days (i.e., social affiliation test with an unfamiliar female followed by vaginal smear).

BrdU administration. BrdU (Sigma) was dissolved in sterile saline at 10 $\mathrm{mg} / \mathrm{ml}$ and administered by intraperitoneal injection at a dose of 100 $\mathrm{mg} / \mathrm{kg}$ every $24 \mathrm{~h}$ for a total of two injections. To assess NSC survival and differentiation during the juvenile period, BrdU was administered on PND 36 and 37 and the mice perfused on PND 72. For assessing NSC survival and differentiation during adulthood, BrdU was administered on PND 97 and 98 and the mice perfused on PND 132.

$c$-fos mapping. Eight juvenile females (PND 27-30) were housed singly and administered BrdU on PND 36 and 37. In adulthood (PND 70), four mice were exposed to an unfamiliar adult female in their home cage for $10 \mathrm{~min}$, while the other four mice were left undisturbed in their home cage. Mice were then perfused $2 \mathrm{~h}$ after the social encounter and processed for immunohistochemistry to detect c-fos expression. To determine the effect of ablating neurogenesis on c-fos activation, we exposed a cohort of 10 juvenile females (TK-GFP, $n=4$; WT, $n=6$; PND 27) and 13 adult mice (TK-GFP, $n=6$; WT, $n=7$; PND 75-95) to intracerebroventricular GCV. After $30 \mathrm{~d}$ of GCV treatment, the mice were exposed to an unfamiliar adult female in their home cage for $10 \mathrm{~min}$ and processed for c-fos mapping as described above.
RNA processing and quantitative PCR. Three female, PND 30 TK-GFP mice were killed by rapid decapitation and the following tissues were dissected and collected under RNAase-free conditions: hippocampus, small intestine, large intestine, liver, spleen, and heart. Approximately 5-10 mg of tissue was obtained and placed in an eppendorf tube, frozen in liquid nitrogen, and stored at $-80^{\circ} \mathrm{C}$ until further use. RNA was harvested from these tissues using TRIzol (Invitrogen) purification followed by DNase treatment (Qiagen) for $30 \mathrm{~min}$ at room temperature followed by RNeasy (Qiagen) cleanup step. One microgram of RNA was used to synthesize cDNA using random primers (Invitrogen) and the superscript III RT kit (Invitrogen). Quantitative PCR was then used to assess levels of TK-GFP in the different tissues using the following primers: GFP-9: CTTCTTCAAGGACGACGGCAACTAC and GFP-10: TTCTGCTTGTCGGCCATGATATAGA to assess levels of TK-GFP; and TBP-F-1: AAAGGGAGAATCATGGACCAGAACAA and TBP-R-1: TGGACTAAAGATGGGAATTCCAGGAG to assess levels of the TATA binding protein (TBP) used as an internal control. Efficiency of all primers was confirmed to be $100 \pm 10 \%$ and the $\Delta \Delta \mathrm{Ct}$ method was used (Pfaffl, 2001) to calculate levels of TK-GFP mRNA in the different tissues with TBP as the internal control and the hippocampus as the comparison group.

Tissue preparation and immunohistochemistry. Mice were anesthetized with pentobarbital $(85 \mathrm{mg} / \mathrm{kg})$ and transcardially perfused with cold $\mathrm{PBS} /$ heparin (50 units/ml) solution followed by $4 \%$ paraformaldehyde. Brains were then postfixed with $4 \%$ paraformaldehyde overnight and equilibrated in a $30 \%$ sucrose solution. Sagittal sections $(40 \mu \mathrm{m})$ were collected from the left hemisphere using a freezing sliding microtome. Every sixth section was pooled to obtain six pools containing a total of 18 slices spaced at $240 \mu \mathrm{m}$ intervals, systematically spanning the entire medial-to-lateral axis of the left hemisphere. Slices processed for BrdU staining were treated with $2 \mathrm{~N} \mathrm{HCl}$ for $30 \mathrm{~min}$ at $37^{\circ} \mathrm{C}$ (this step was skipped for slices singly stained with all other primary antibodies) followed by $2 \% \mathrm{H}_{2} \mathrm{O}_{2}$ and a 30 min blocking step with TBST [ $20 \mathrm{~mm}$ Tris- $\mathrm{Cl}$ ( $\mathrm{pH}$ 7.4), $150 \mathrm{~mm} \mathrm{NaCl}, 0.3 \%$ Triton X-100] containing $10 \%$ horse serum (The Jackson Laboratory). Slices were then incubated overnight at $4^{\circ} \mathrm{C}$ with mouse anti-BrdU (1:100; catalog no. 347580 ; Becton Dickinson), goat anti-doublecortin antibodies (1:500; catalog no. sc-8066; Santa Cruz Biotechnology), rabbit anti-c-fos antibodies (ab-5, 1:4000; catalog no. pc 38; Calbiochem), mouse anti-GFAP (1:200; catalog no. G 3893; Sigma), rat anti-mouse CD68 (1:100; catalog no. MCA1957; ABD Serotect), or rabbit anti-Ibal (1:250; catalog no. 019-19741; Wako), followed by incubation with the appropriate secondary biotinylated antibodies (Vector Labs). ABC kit (Vector Labs) was used to amplify the signal followed by staining with $3^{\prime}, 3^{\prime}$ diaminobenzidine (DAB). Slices were then dried overnight and counterstained with Nuclear Fast stain (Vector Laboratories). For experiments involving colocalization of BrdU with other cellular markers such as GFP or c-fos, tissue was first treated with 10,000 U DNase (Worthington Biochemicals) in TBST plus $1 \mathrm{~mm}$ $\mathrm{MgCl}_{2}$ for $30 \mathrm{~min}$ at room temperature followed by $24-48 \mathrm{~h}$ incubation with rabbit anti-GFP (1:5000; catalog no. ab290; Abcam) or rabbit antic-fos antibodies (ab-5; 1:2000; catalog no. pc 38; Calbiochem) and mouse anti-BrdU as above. A similar protocol was used to assess colocalization of BrdU and mouse anti-NeuN antibodies (1:1000; catalog no. MAB377; Millipore) except that anti-rat BrdU antibodies were used (1:100; catalog no. OBT 0030; Accurate Chemical). All secondary antibodies were from Invitrogen.

Stereology. Stereological studies were conducted in the DG, MOB, and corpus callosum, and followed previously reported protocol (Shors et al., 2001), with the following modifications: Stereo Investigator 8 software (MBF Bioscience) was used in all counting experiments, with borders of these brain regions defined under low magnification $(2.5 \times)$. Grid and counting frame sizes were designed to count a total of 500-1000 cells in each brain region of a wild-type animal. Total number of cells in the left hemisphere $\left(N_{\mathrm{L}}\right)$ was then estimated as $N_{\mathrm{L}}=\Sigma \mathrm{Q}^{-}{ }^{\star} 1 / \mathrm{ssf}^{\star} 1 /$ asf ${ }^{\star} 1 / \mathrm{tsf}$, where ssf is the sampling fraction (1/6 in our case) and asf (area sampling fraction) $=$ area of counting frame/area of grid. This ratio ranges from 1 to 16 depending on the brain region and the cellular marker counted. In accordance with the modified version of the optical fractionator method, the tsf (thickness sampling fraction) was assumed to be 1 (Shors et al., 2001). $N_{\mathrm{L}}$ was then doubled to estimate the number of cells in both 
hemispheres. Counting was done under $40 \times$ magnification by an observer who was blind to the genotype of the mouse and followed conventional principles of the optical disector method. The counting/recounting reliability of this method was $r=0.93, p=0.02$.

Microscopy. High-resolution $(1024 \times 1024$ pixels $)$ confocal images were obtained using a Zeiss LSM 510 Meta microscope under a $63 \times$ apochromat water-immersion lens (1.2 NA). Bright microscopy and stereological studies were conducted using an Axioskop I (Zeiss) microscope equipped with a motorized stage, a CCD camera connected to a large monitor, and a computer loaded with Stereo Investigator 8.0 software. For confocal studies that assessed the percentage of colocalization between BrdU-positive cells and c-fos-positive cells in the MOB and DG of mice exposed to social encounter or kept isolated, BrdU cells $(n=50-70$ per animal; $n=4$ mice per group) were randomly selected using at least six different slices per animal and colocalization with c-fos was determined under a $63 \times$ apochromat water-immersion lens $(1.2 \mathrm{NA})$. A similar procedure was used to assess the percentage of BrdU cells and c-fos-positive cells that coexpressed the mature neuronal marker NeuN.

Statistical analysis. Data were carefully screened for inaccuracies, outliers, normality, and homogeneity of variance. Unpaired Student's $t$ tests were used in cases when only two independent groups were analyzed, with the exception of the pup retrieval test, where $\chi^{2}$ analysis that was used. Replicability and validity of results were verified by using two different age groups and retesting them using additional independent cohorts of mice. A $2 \times 2$ ANOVA was used to assess the effects of route of GCV administration (intracerebroventricular vs subcutaneous) and genotype (TK-GFP vs WT) on weight gain followed by simple effect analyses for each genotype. Bivariate Pearson analysis was used to assess the correlation between the log number of BrdU-positive cells and social behavior. Repeated-measure ANOVA was used to analyze the effects of social encounter or genotype as between-subject variables and brain region as a within-subject variable on c-fos expression. Significant between-subject effects were followed by simple-effect analysis for each brain region. Repeated-measure ANOVA was used to assess the effects of day of testing as a within-subject variable and group (high vs low social score on day 1) as a between-subject variable on social exploration as the dependent variable. For developmental studies, one-way ANOVA was used to assess the main effect of experimental group on social exploration, escape behavior, the latency to find food, and exploration of inanimate toy. One-way ANOVA was also used to assess the main effect of estrus phase (e.g., proestrus, estrus, metestrus, diestrus) on social behavior. Significant main effects were followed by Tukey-HSD post hoc analysis.

\section{Results}

Direct administration of GCV into the lateral ventricle eliminates neurogenesis in an efficient and nontoxic manner Staining brain tissue from juvenile mice (PND 30-40) with antibodies that label immature neurons (i.e., anti-doublecortin antibodies) confirmed previous reports (Hatten, 1999; Lemasson et al., 2005; Li et al., 2009) showing that neurogenesis at this age, like that of adult neurogenesis, is mainly confined to the SVZ-MOB and the SGZ of the DG (data not shown). Cell counting of doublecortin-positive cells (DCX) showed that levels of neurogenesis are tenfold higher in the $\operatorname{MOB}\left(t_{(8)}=8.11, p<0.013\right)$ and fourfold higher in the DG $\left(t_{(8)}=10.76, p<0.0005\right)$ in juvenile (i.e., PND 30-40, $n=5$ ) compared with adult (PND 90-120, $n=5$ ) mice. These studies confirm previous work showing an age-dependent decrease in levels of neurogenesis during postnatal development in rodents and nonhuman primates (Altman and Das, 1965; Pencea et al., 2001; Lemasson et al., 2005).

The observation that juvenile neurogenesis resembles that of adult mice in its anatomical distribution, yet is driven at much greater rates, raised the question as to whether neurogenesis during these two periods serves similar or distinct functions in guiding complex behaviors in adulthood. To address this issue, we developed a method to block NSC proliferation in a rapid, cell- specific, and nontoxic manner. This was done by generating transgenic mice that express TK-GFP in NSCs (Fig. 1A). Among the several founders obtained, we chose a line that showed restrictive expression of GFP in the SVZ (Fig. 1B) and SGZ (Fig. 1C). Similar patterns of GFP expression were seen in juvenile and adult mice, with no GFP detected in the cortex, corpus callosum, cerebellum, amygdala, spinal cord, or hypothalamus. Moreover, TK-GFP protein was colocalized with known markers of NSC such as GFAP and nestin. Labeling mitotically active cells with BrdU showed that $44 \pm 4 \%$ of the dividing cells also expressed GFP. Analysis of different tissues revealed that TK-GFP mRNA was also expressed in the intestine and heart (Fig. $1 D$ ), a pattern that is consistent with previous reports using the nestin promoter (Zimmerman et al., 1994; Bush et al., 1998).

The non-CNS expression of TK-GFP and the relatively poor ability of GCV to cross the blood-brain barrier (Brewster et al., 1994) raised concerns about possible peripheral toxicity associated with systemic administration of GCV. Indeed, $30 \mathrm{~d}$ administration of GCV via osmotic minipumps implanted subcutaneously required high dose of GCV (i.e., $100 \mathrm{mg} / \mathrm{kg}$ ) to block neurogenesis, was associated with poor coat condition, ulcerative blepharitis (i.e., erythematous lesions around the eyes), and weight loss $(-3.5 \pm 1.5 \mathrm{~g})$, confirming previous reports (Bush et al., 1998; Singer et al., 2009). In contrast, TK-GFP mice exposed to continuous intracerebroventricular administration of GCV $(0.26 \mathrm{mg} / \mathrm{d})$ for the same duration showed normal weight gain $(+1.5 \pm 0.48 \mathrm{~g})$ and no signs of peripheral toxicity $\left(F_{(1,14)}=9.86, p=0.007\right.$, for a simple-effect analysis comparing weight gain in TK-GFP mice exposed to subcutaneous vs intracerebroventricular GCV).

Intracerebroventricular administration of GCV eliminated DCX-positive cells in DG and MOB (Fig. $1 E-J$ ) without inducing reactive gliosis (Fig. $1 \mathrm{~K}$ ) or inflammatory response (Fig. $1 L$ ). Numbers of DCX-positive cells were similar in wild-type females exposed to intracerebroventricular GCV $(n=5)$, wild-type females exposed to PBS $(n=4)$, and TK-GFP littermates exposed to intracerebroventricular PBS $(n=5)$, demonstrating that rates of neurogenesis were similar in TK-GFP and wild-type mice, and that exposure to GCV did not modify levels of neurogenesis in wild-type mice (DG: $F_{(2,12)}=0.7, p=0.51 ; \mathrm{MOB}: F_{(2,12)}=0.89$, $p=0.43$; one-way ANOVA). Together, these studies show that intracerebroventricular GCV provides a rapid and efficient method for eliminating neurogenesis in a manner that is not associated with peripheral toxicity, reactive gliosis, or inflammatory response.

\section{Exposure to GCV eliminates greater number of neurons during the juvenile period compared with adulthood}

To assess the effects of $30 \mathrm{~d}$ exposure of GCV delivered intracerebroventricularly on NSC survival in juvenile and adult animals, we exposed a cohort of juvenile TK-GFP and wild-type female littermates (PND 27-35, $n=7$ for each genotype) and a second cohort of age-matched adult female littermates (PND 90-120, $n=6-7$ for each genotype) to $30 \mathrm{~d}$ of intracerebroventricular GCV. After surgeries, all females were singly housed and were administered BrdU (100 mg/kg) 7 and $8 \mathrm{~d}$ postsurgery to compare rates of NSC survival at these two ages. Behavior was assessed in adulthood after which the mice were perfused to assess the relative effect of intracerebroventricular GCV on NSC survival (for schematic timelines, see Fig. 2A,E).

Exposure to GCV during both the juvenile and adult periods eliminated $90-95 \%$ of the DCX-positive cells (data not shown) and BrdU-positive cells in the MOB (Fig. 2B,F) and DG (Fig. $2 C, G)$, demonstrating similarly rapid and efficient blockade of 

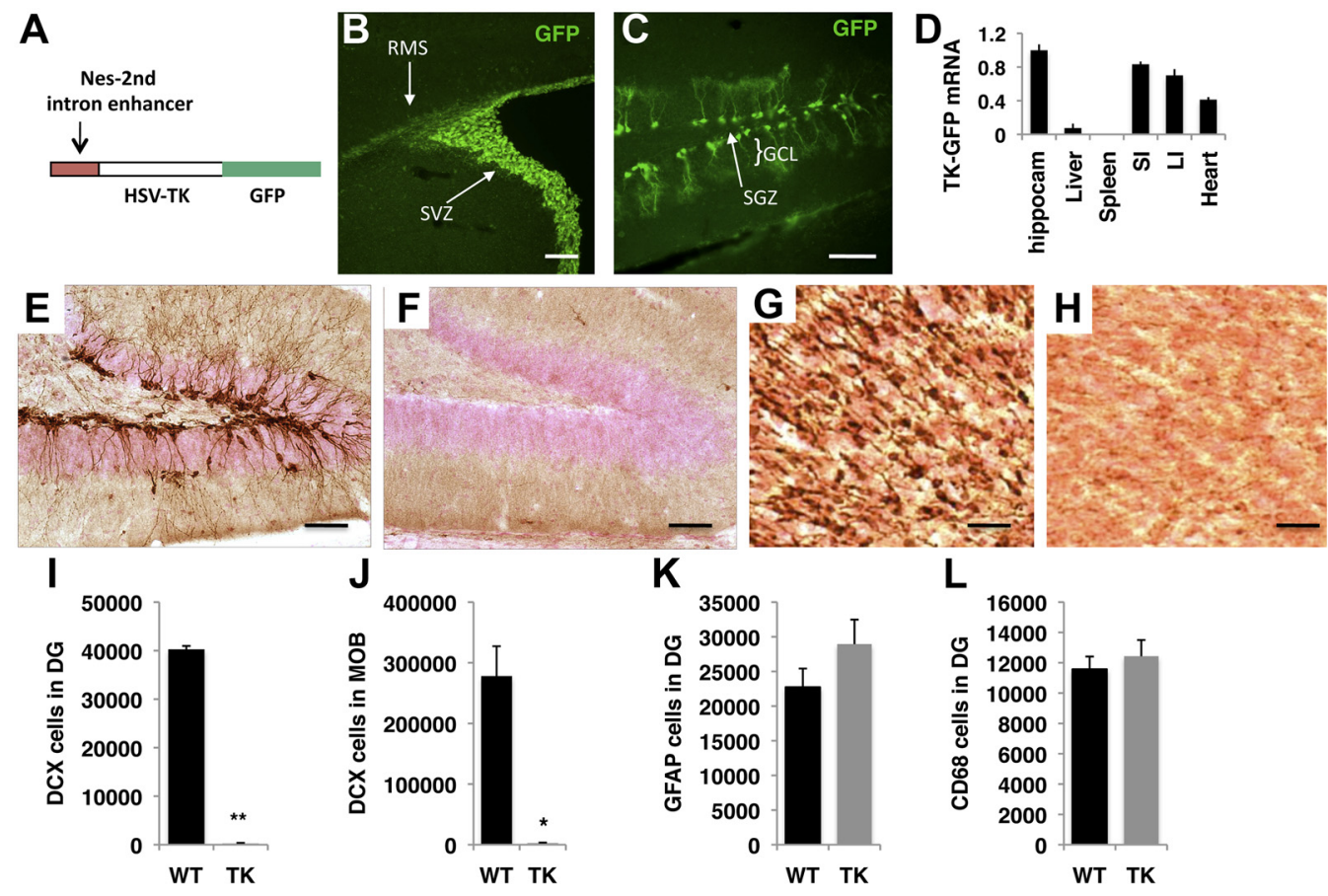

Figure 1. Intracerebroventricular delivery of GCV efficiently eliminates neurogenesis in TK-GFP transgenic mice without causing gliosis or inflammatory response. $A$, Construct used to generate TK-GFP mice. $\boldsymbol{B}, \boldsymbol{C}$, Expression of TK-GFP (green) is restricted to the SVZ and the rostral migratory stream (B) and SGZ of the DG (C). D, Quantitative PCR showing relative mRNA levels of TK-GFP in different tissues. $\boldsymbol{E}$ - L, WT and TK-GFP littermate females ( $n=3$ for each genotype) were exposed to GCV delivered intracerebroventricularly on PND $30-70$ and perfused on PND 70. Representative pictures showing levels of immature neurons stained with anti-DCX antibodies (dark brown) in the DG $(\boldsymbol{E}, \boldsymbol{F})$ and MOB $(\boldsymbol{G}, \boldsymbol{H})$ of WT $(\boldsymbol{E}, \boldsymbol{G})$ and TK-GFP $(\boldsymbol{F}, \boldsymbol{H})$ females. This protocol eliminates $95 \%$ of the DCX-positive cells in the DG $(I)$ and MOB $(\boldsymbol{J})$ without modifying numbers of GFAP-positive cells (a marker of gliosis; $K$ ) or number of CD68-positive cells (a marker of activated microglia cells; L). GFAP- and CD68-postive cells were counted throughout the entire DG and similar results were obtained when GCV was administered intracerebroventricularly in adulthood. Nes, Nestin; HSV-TK, TK from the Herpes simplex virus; SI, small intestine; LI, large intestine; RMS, rostral migratory stream; GCL, granular cell layer. Error bars represent mean $\pm S E M$. ${ }^{*} p<0.05$, ${ }^{* *} p<0.01$, unpaired Student's $t$ test. Scale bars, $50 \mu \mathrm{m}$.
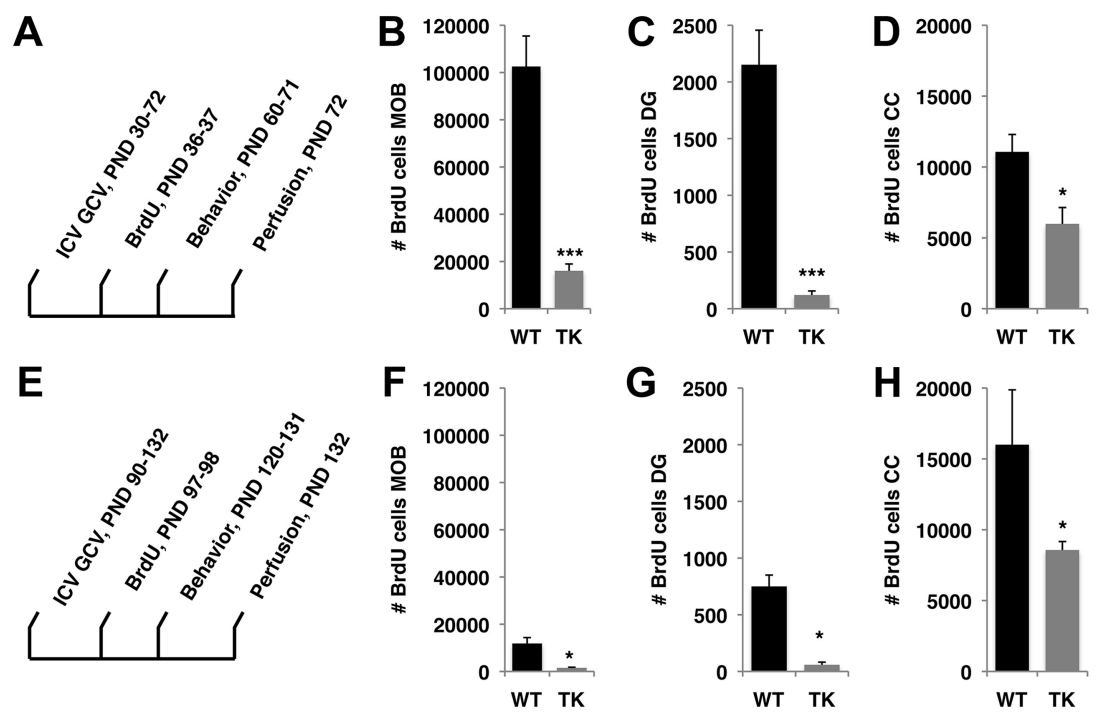

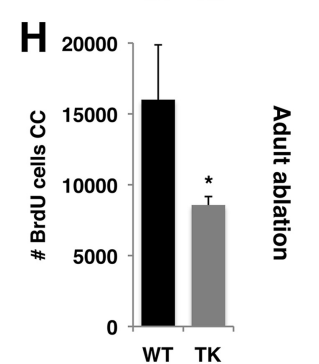

Figure 2. $\boldsymbol{A}-\boldsymbol{H}$, Exposure to $\mathrm{GCV}$ eliminates greater number of neurons during the juvenile period $(\boldsymbol{A}-\boldsymbol{D})$ compared with adulthood $(\boldsymbol{E}-\boldsymbol{H}) . \boldsymbol{A}, \boldsymbol{E}$, Schematic timelines for the juvenile-ablation $(\boldsymbol{A})$ and adult-ablation $(\boldsymbol{E})$ cohorts. $\boldsymbol{B}, \boldsymbol{C}, \boldsymbol{F}, \boldsymbol{G}$, Exposure to $\mathrm{GCV}$ during the juvenile period eliminated significantly more BrdU-positive cells in the MOB $(\boldsymbol{B}, \boldsymbol{F})$ and $D G(\boldsymbol{C}, \boldsymbol{G})$ compared with adulthood. $\boldsymbol{D}, \boldsymbol{H}$, Exposure to $\mathrm{GCV}$ in the juvenile period and adulthood eliminated similar numbers of non-neuronal BrdU-positive cells in the corpus callosum. Same $y$-axis scales were used for each brain region to emphasize scope of NSC elimination in these two age groups. Error bars represent mean \pm SEM. ${ }^{*} p<0.05,{ }^{* * *} p<0.001$, unpaired Student's $t$ test. ICV, Intracerebroventricular.

NSC survival in both age groups (note that BrdU was administered 7 and $8 \mathrm{~d}$ after the intracerebroventricular surgeries in both groups; Fig. $2 A, E)$. At both ages, $>90 \%$ of the BrdU cells in the MOB and DG expressed the NeuN marker, indicating that the majority of BrdU cells eliminated in the DG and $\mathrm{MOB}$ were neurons. As expected, the absolute numbers of neurons eliminated during the juvenile period in the MOB and DG were significantly greater compared with those eliminated in adulthood (compare Fig. 2, $B$ to $F$ and $C$ to $G$ ). Females exposed to intracerebroventricular GCV during the juvenile period gained significantly more weight than adult females (juvenile: $+7.7 \pm 0.23 \mathrm{~g}$; adult: $+2.1 \pm 0.34 \mathrm{~g}$ ), consistent with the rapid growth associated with this developmental phase. However, in both age groups, TK-GFP and wild-type females showed no signs of peripheral toxicity and gained similar amounts of weights $(p>0.05)$, indicating that exposure to GCV delivered intracerebroventricularly was well tolerated at both ages.

Since nestin-positive NSC are able to differentiate into non-neuronal lineages such as oligodendrocytes and astrocytes (Doetsch and Hen, 2005), we assessed the relative effects of GCV exposure on nonneuronal lineages. To accomplish this, we took advantage of previous work showing that the corpus callosum contains a large number of non-neural BrdU-positive cells (Cerghet et al., 2006) and confirmed that none of the BrdU-positive cells found in the corpus callosum expressed the neuronal marker NeuN (data not 
shown). Next, we compared the effect of GCV exposure in juvenile and adult animals on survival of BrdU-positive cells in the corpus callosum. This analysis showed that exposure to GCV caused a 50\% reduction in the number of BrdU-positive cells in both ages (Fig. 2D,H), demonstrating that GCV treatment was not specific to neuronal lineages. However, unlike the significantly greater numbers of BrdU-positive cells eliminated in the MOB and DG during the juvenile period, similar numbers of BrdU-positive cells were eliminated in the corpus callosum of juvenile and adult mice $\left(t_{(12)}=1.11, p=0.28\right)$.

\section{Ablating juvenile and adult neurogenesis causes similar behavioral outcomes in tests of anxiety and memory \\ Ablating neurogenesis in both ages had no effect on time exploring the center (Fig. $3 A, F$ ) or the distance traveled (Fig. $3 B, G$ )} in the open-field test. Blocking neurogenesis in both age groups increased time in the illuminated area (juveniles: $t_{(12)}=2.68, p=0.020$; Fig. $3 C$; adults: $t_{(11)}=2.21, p=$ 0.049 ; Fig. $3 H$ ) and number of transitions in the dark-light assay (juveniles: $t_{(12)}=2.40, p=0.033$; Fig. $3 D$; adults: $t_{(11)}=2.52, p=$ 0.028; Fig. $3 I$ ), indicating decreased anxiety-like behavior. Exposure to GCV in both ages eliminated the normal tendency to explore a novel object (juveniles: $t_{(12)}=2.31, p=0.039$; Fig. $3 E$; adults $t_{(11)}=2.22, p=0.048$; Fig. $\left.3 J\right)$, consistent with impaired hippocampal-function in these mice. There were no differences between TK-GFP and wild-type mice in the distance traveled or the amount of time these mice investigated objects during the training session $(p>0.05)$, suggesting that the failure of TK-GFP to preferentially explore the novel object was not due to inadequate object exploration during the training session. TK-GFP and wild-type mice that were not exposed to GCV showed no differences in these behaviors (Table 1), indicating that exposure to GCV is necessary to induce these behavioral changes. Together, these data demonstrate that neurogenesis during both periods share some common functions.

\section{Juvenile but not adult neurogenesis is required for normal social behavior}

Next, we examined the role of neurogenesis in female-female social interaction. We chose female-female rather than femalemale social interaction for several reasons. First, previous work suggested that female-female social exploration plays a role in functions that are important for maintaining stable social networks (Maggio and Whitney, 1985) and reproduction (Drickamer, 1974). Second, females spend significantly more time exploring unfamiliar females compared with males (100.5 \pm $17.0 \mathrm{~s}$ vs $40.5 \pm 12.8 \mathrm{~s}$, respectively, $p=0.018$ ). Third, unlike interactions with males that are highly influenced by the estrus cycle (Thornton and Finn, 1999; Barthelemy et al., 2004), social interaction with other females is not affected by the estrus cycle $\left(F_{(3,23)}=0.433, p=0.73\right.$, one-way ANOVA $)$. Finally, repeated testing over a course of $4 \mathrm{~d}$ showed that individuals that displayed high levels of social interaction on the first day of testing continued to maintain higher levels of social interaction on days 2-4 compared with those showing low levels of interactions on the
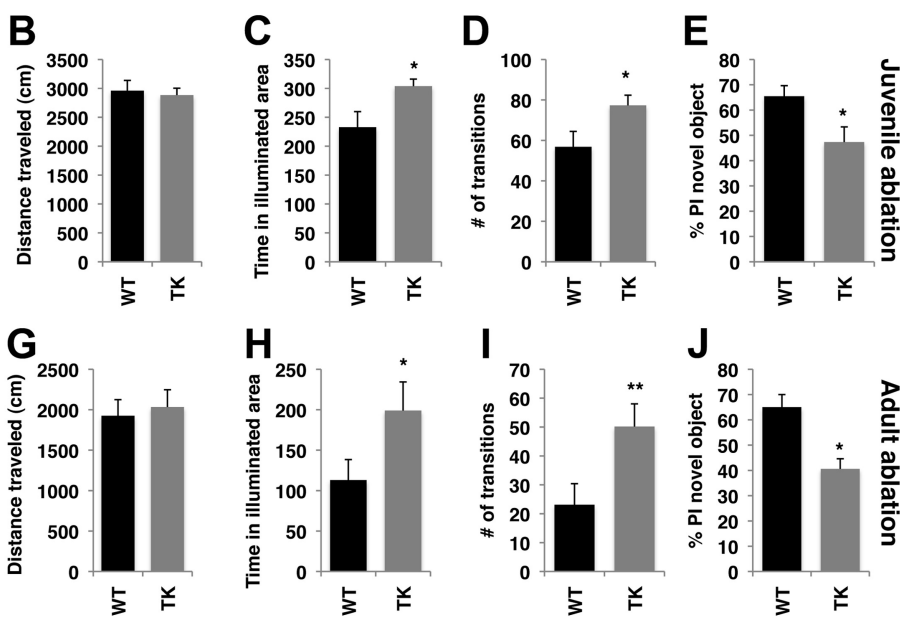

Table 1. Behavioral characterization of TK-GFP and wild-type adult females

\begin{tabular}{lccc}
\hline Behavior & $\begin{array}{c}\text { WT, mean } \pm \text { SEM } \\
(n=10)\end{array}$ & $\begin{array}{l}\text { TK-GFP, mean } \pm \text { SEM } \\
(n=8)\end{array}$ & $\begin{array}{l}p \\
\text { value }\end{array}$ \\
\hline Time in the center (s), open field test & $11.2 \pm 1.1$ & $9.0 \pm 1.3$ & 0.20 \\
Distance traveled (cm), open field test & $2030 \pm 88$ & $1978 \pm 116$ & 0.72 \\
$\begin{array}{l}\text { Time in the illuminated area (s), } \\
\quad \text { dark-light test }\end{array}$ & $172 \pm 38$ & $176 \pm 42$ & 0.95 \\
$\begin{array}{l}\text { Number of transitions, dark-light test } \\
\text { Preference index to novel object (\%), } \\
\quad \text { object-recognition task }\end{array}$ & $37.2 \pm 7.9$ & $38.7 \pm 8.0$ & 0.90 \\
$\begin{array}{l}\text { Time exploring object mouse (s), } \\
\text { social affiliation test }\end{array}$ & $77.9 \pm 5.1$ & $84.9 \pm 3.2$ & 0.30 \\
$\begin{array}{l}\text { Number of escapes, social affiliation } \\
\text { test }\end{array}$ & $0.8 \pm 0.25$ & $1.37 \pm 0.42$ & 0.43 \\
\hline
\end{tabular}

Wild-type $(n=10)$ and TK-GFP $(n=8)$ littermates $(n=10)$ were singly housed from PND 30 - 65 and tested in the following behaviors from PND 66 -72: open-field, dark-light, object-recognition, and social affiliation test. These mice did not undergo intracerebroventricular surgery or exposure to GCV. However, exposure to intracerebroventricularly delivered PBS during this same period did not modify social behavior in TK-GFP mice (Fig. 7A,B), indicating that exposure to intracerebroventricularly delivered GCV is necessary to induce social abnormalities in TK-GFP female mice.

first day $\left(F_{(1,8)}=13.29, p=0.007\right.$, between-group effect), indicating that the tendency to explore an unfamiliar female is a stable behavioral trait.

Surprisingly, blocking juvenile neurogenesis substituted the normal tendency of female mice to socially explore other females with social aversion that included a nearly complete absence of social exploration $\left(t_{(12)}=4.40, p<0.0005\right.$; Fig. $\left.4 A\right)$ or time maintaining close proximity with a conspecific $\left(t_{(12)}=5.07, p<\right.$ 0.0005; Fig. 4B). Moreover, TK-GFP females exposed to GCV during the juvenile period evaded any attempts made by the conspecific to engage in social behavior $\left(t_{(12)}=4.79, p<0.0005\right.$; Fig. $4 C)$. This escape behavior is clearly related to social cues because the same mice showed fewer anxiety-like behaviors compared with wild-type mice in a nonsocial dark-light test (Fig. 3C,D).

We subjected the mice to a pup-retrieval test by randomly placing three newborn pups in the mice's home cage. Nulliparous female mice are spontaneously maternal and social cues emitted by the pups normally elicit maternal retrieval and gathering of pups with attempts to nurse in the nest. As shown in Figure $4 D$, 

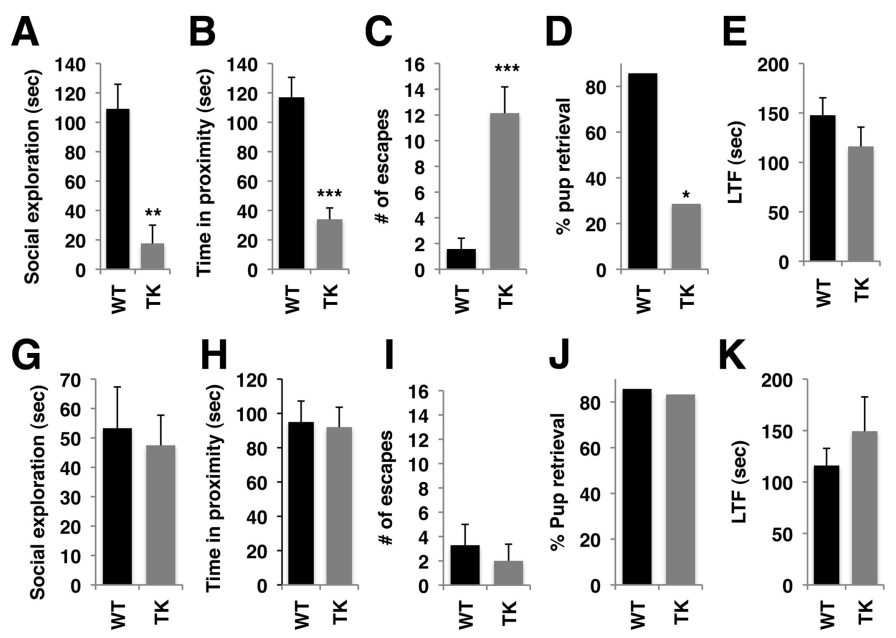

Figure 4. Ablating juvenile, but not adult, neurogenesis causes profound social deficits in adulthood. $A-L$, Behavioral testing of social behaviors and olfactory function was conducted after juvenile $(\boldsymbol{A}-\boldsymbol{F})$ and adult $(\boldsymbol{G}-\boldsymbol{L})$ ablation of neurogenesis. Ablating neurogenesis during the juvenile period, but not in adulthood, decreased social exploration $(\boldsymbol{A}, \boldsymbol{G})$, decreased time in close proximity with a conspecific $(\boldsymbol{B}, \boldsymbol{H})$, increased escape behavior $(\boldsymbol{C}, \boldsymbol{I})$, and impaired pup retrieval $(\boldsymbol{D}, \boldsymbol{J})$. Ablation of neurogenesis in both ages had no effect on the latency to find food $(\mathrm{LTF} ; \boldsymbol{E}, \boldsymbol{K})$ or the ability to discriminate between urine and water $(\boldsymbol{F}, \boldsymbol{L})$. Error bars represent mean \pm SEM. ${ }^{*} p<0.05,{ }^{* *} p<0.01,{ }^{* * *} p<0.001$, unpaired Student's $t$ test in all panels except $\boldsymbol{D}$ and $\boldsymbol{I}$, where $\chi^{2}$ analysis was done.
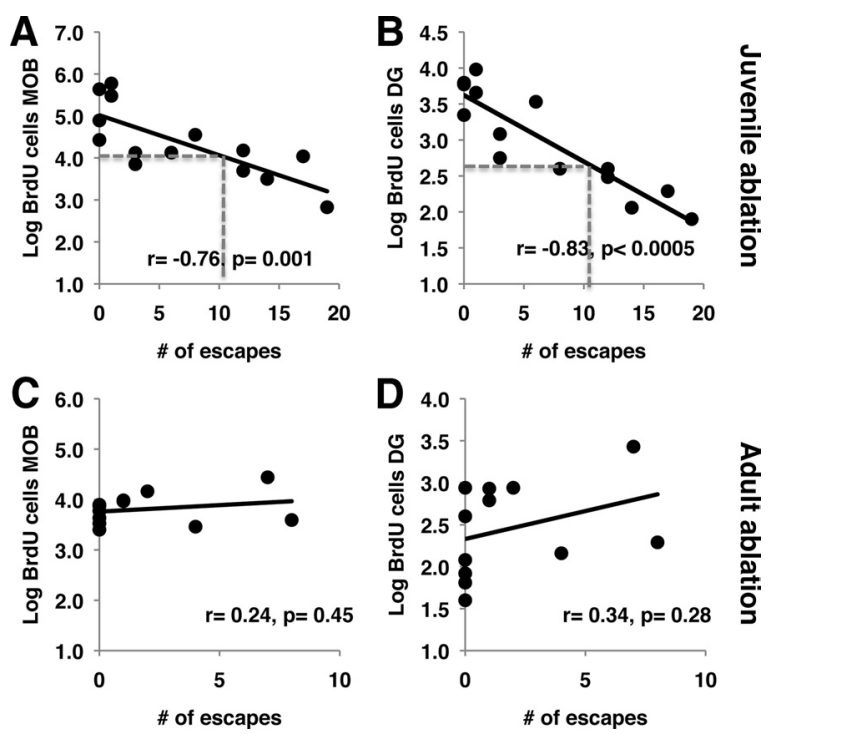

Figure 5. Juvenile, but not adult, neurogenesis predicts adult social behavior. $A-D$, Escape behavior in adulthood was highly correlated with the log number ofBrdU-positive cells generated during the juvenile period $(\boldsymbol{A}, \boldsymbol{B})$ but not in adulthood $(\boldsymbol{C}, \boldsymbol{D})$ in the $\operatorname{MOB}(\boldsymbol{A}, \boldsymbol{C})$ and $D G(\boldsymbol{B}, \boldsymbol{D})$. Note that these are the same animals used in Figures $2-4$. The dotted lines in $\boldsymbol{A}$ and $\boldsymbol{B}$ show the predicted escape behavior for an animal in which juvenile neurogenesis was reduced to levels seen in a normal adult female. Note that such an animal will show an average of 10 escapes in adulthood.

nearly $90 \%$ of the wild-type females gathered and huddled over the pups within $15 \mathrm{~min}$ as opposed to only $30 \%$ of the TK-GFP female mice exposed to intracerebroventricular GCV during the juvenile period $\left(\chi^{2}=4.67, p=0.031\right)$. Ablation of juvenile neurogenesis had no affect on the ability of these mice to locate a small pellet of food hidden under $2 \mathrm{~cm}$ of bedding ( $p>0.05$; Fig. $4 E)$ or to discriminate between urine and water $(p>0.05$; Fig. $4 F$ ), demonstrating that juvenile ablation of neurogenesis does not cause anosmia.

Eliminating neurogenesis in adult mice had no effect on any of the social behaviors tested (Fig. $4 G-J$ ), indicating that juvenile

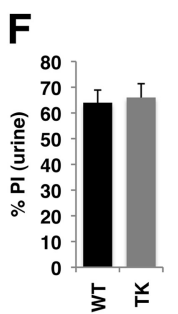

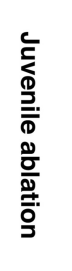

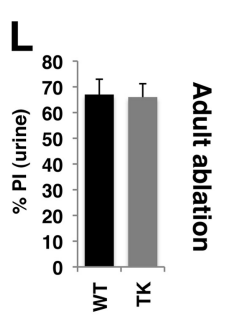

and adult neurogenesis make different contributions to social competency in adulthood. Adult ablation of neurogenesis had no effect on the latency to find food ( $p>0.05$; Fig. $4 K$ ) or the ability to discriminate between urine and water $(p>0.05$; Fig. $4 L)$, consistent with previous work showing that olfactory function is grossly intact after ablating adult neurogenesis in the MOB (Mak et al., 2007; Imayoshi et al., 2008; Breton-Provencher et al., 2009; Lazarini et al., 2009; Valley et al., 2009). Social behaviors in PND 70 and PND 130 wild-type mice were similar, indicating that differences in social outcomes were unlikely to be explained by testing these behaviors at somewhat different ages. Moreover, the normal social behaviors seen in mice with intact neurogenesis or those with adult ablation of neurogenesis indicate that manipulations such as intracerebroventricular surgery, exposure to GCV, and single housing the mice at both ages are not sufficient to induce abnormal social behavior in adulthood.

Interestingly, escape behavior in adulthood was highly correlated with the log number of BrdU-labeled cells generated during the juvenile period in the MOB (Fig. 5A) and DG (Fig. 5B) and significant correlations were also seen when the data were analyzed separately for wild-type and TK-GFP animals (data not shown). These findings indicate that blocking juvenile neurogenesis explains approximately half of the variance in the escape behavior shown in Figure 4C. Similar correlations were found between the amount of time females spent in social exploration and the log number of BrdU cells generated during the juvenile in the MOB $(r=+0.67, p=0.0009)$ and DG $(r=+0.61, p=0.02)$. In contrast, labeling NSC with BrdU during adulthood showed no correlation with escape behavior in adulthood (Fig. 5C,D), consistent with the notion that levels of neurogenesis during the juvenile period, but not in adulthood, plays an important role in guiding social competency in adulthood. These data also suggest that high levels of neurogenesis during the juvenile period are necessary to support normal social behavior in adulthood, and that decreasing juvenile neurogenesis to levels seen in adulthood (Fig. $5 C, D$ ) would give rise to a female with high social phobia in adulthood (Fig. $5 A, B$, dotted lines).

Social encounter induces c-fos activation in the MOB and DG in a manner that requires juvenile neurogenesis

Next, we used c-fos mapping in adult mice and showed that a brief social interaction with a conspecific induces expression of this immediate early gene in several brain regions, most notably the $\mathrm{MOB}$, the hippocampus, and several cortical areas (Fig. 6A, B). Consistent with previous work, $96 \pm 3 \%$ of these c-fos-positive cells in the MOB were mature neurons based on colocalization of c-fos with the neuronal marker NeuN (Magavi et al., 2005). We speculated that substantial numbers of these social response neurons were generated during the juvenile period. We tested this hypothesis by administrating BrdU to singly housed juvenile female mice (PND 36, 37), exposing them to a conspecific in their home cage for $10 \mathrm{~min}$ when they reached adulthood (PND 70), and perfusing them $2 \mathrm{~h}$ after social exposure. As expected, the majority of the BrdU cells ( $92 \pm 3 \%$ ) labeled during the juvenile 


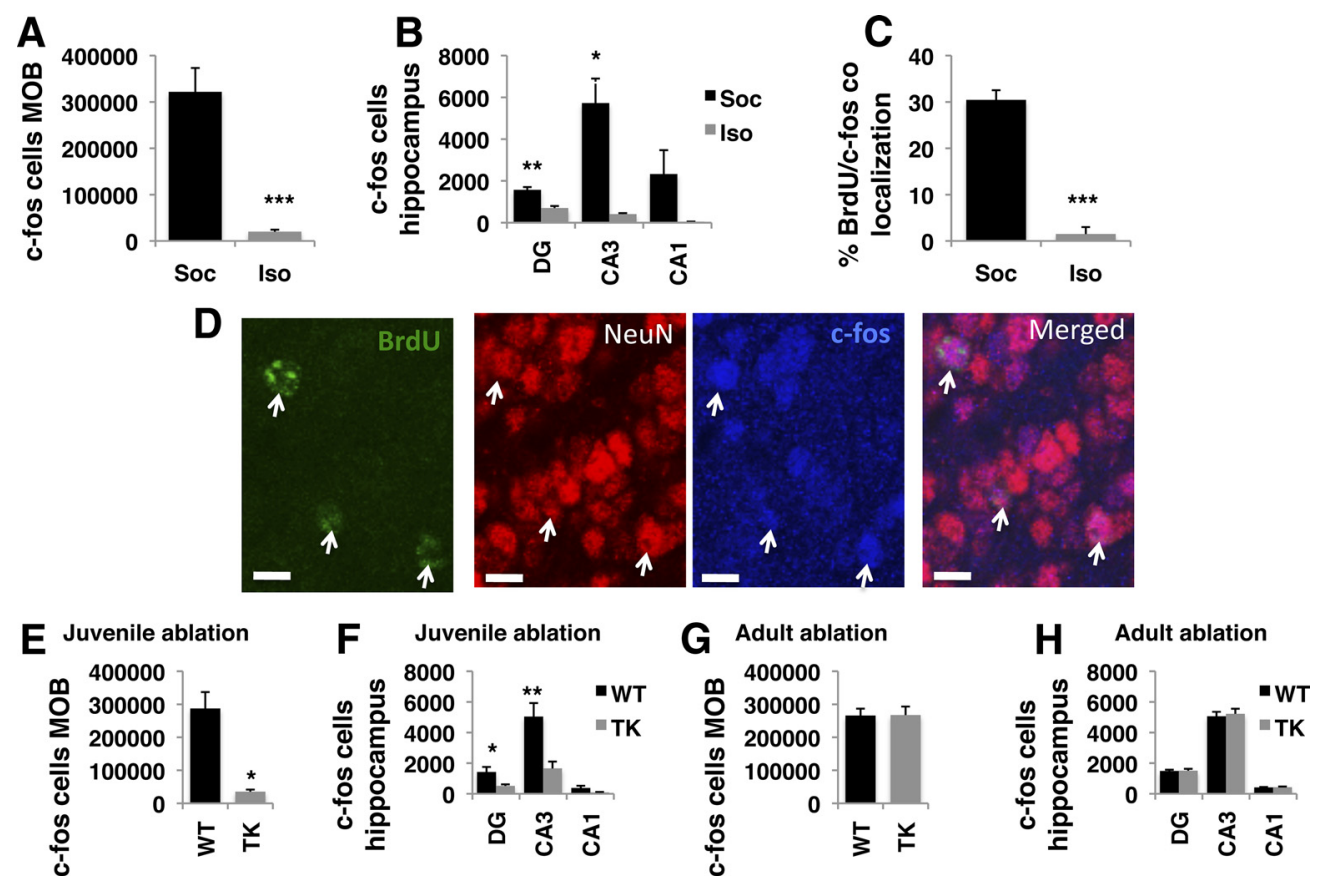

Figure 6. Social encounter induces c-fos activation in the MOB and DG in a manner that requires juvenile neurogenesis. $A$, Number of c-fos-positive cells in the MOB increased in wild-type mice exposed to a conspecific in their home cage $(S o c, n=4)$ compared with mice left socially isolated (Iso, $n=4)$. B, Social encounter also increased the number of c-fos-positive cells in all subfields of the hippocampus. C, Approximately $30 \%$ of NSC labeled with BrdU on PND 36 and 37 induced c-fos expression in the MOB in response to a social encounter. D, Pictures of MOB showing that BrdU cells (green) labeled on PND 36 and 37 express NeuN (red) and induce expression of c-fos (blue) in response to a social encounter in adulthood. $\boldsymbol{E}-\boldsymbol{H}$, Intracerebroventricular GCV treatment for $30 \mathrm{~d}$ during the juvenile period $\left(F_{(1,8)}=18.5, p=0.0026\right.$, repeated-measure ANOVA) but not in adulthood decreased c-fos activation in response to a brief social encounter in the MOB $(\boldsymbol{E}, \boldsymbol{G})$ and hippocampus $(\boldsymbol{F}, \boldsymbol{H})$. Error bars represent mean \pm SEM. ${ }^{*} p<0.05,{ }^{* *} p<0.01,{ }^{* * *} p<0.005$. Scale bars, $10 \mu \mathrm{m}$.

period expressed the NeuN marker, indicating that most of these cells differentiated into neurons in adulthood. Importantly, 30\% of the BrdU-labeled cells in the MOB expressed c-fos in response to a social encounter $(n=278$; Fig. $6 C, D)$. This was not the case with BrdU-labeled cells in the DG, where none of the BrdUpositive cells examined $(n=256)$ expressed c-fos. These data suggest that both the hippocampus and the MOB are activated in response to a social encounter in adulthood, and that a substantial portion of the neurons activated in the MOB were generated during the juvenile period.

To test whether juvenile neurogenesis is necessary to induce c-fos expression, we ablated juvenile neurogenesis and assessed c-fos activation after a brief social encounter in adulthood. Wildtype mice showed intense social exploration in their home cage that was associated with robust induction of c-fos expression in the MOB and hippocampus, demonstrating that exposure to intracerebroventricular GCV during the juvenile period had no effect on c-fos activation in these mice. In contrast, TK-GFP mice evaded social contact in their home cage, a behavioral response that was associated with no $c$-fos induction in the MOB and the hippocampus $\left(F_{(1,8)}=18.5, p=0.0026\right.$; Fig. $\left.6 E, F\right)$. These findings demonstrate that juvenile neurogenesis is required for the induction of c-fos in response to brief social exposure in adulthood. Levels of c-fos activation in the MOB and DG in mice with juvenile ablation of neurogenesis were similar to those seen in intact mice that were kept socially isolated $\left(F_{(1,6)}=3.69, p=\right.$ 0.103 ; compare Fig. $6, A$ to $E$ and $B$ to $F$ ), suggesting that in the absence of active social investigation, these mice were unable to detect social cues present in their home cage. Ablation of neurogenesis in adult mice had no affect on c-fos activation in response to a brief social encounter $\left(F_{(1,11)}=0.003, p=0.96\right.$; Fig. $6 G, H)$, consistent with the notion that adult neurogenesis is not required to support appropriate processing of socially mediated cues.

Social behavior matures during the juvenile period in a manner that requires juvenile neurogenesis

We predicted that if juvenile neurogenesis is required for maturation of adult-like social behavior, we should see a gradual shift from social aversion to social interest during this period. Indeed, social interaction increased during the juvenile period and reached an adult-like phenotype at around PND $55\left(F_{(4,32)}=\right.$ 123.8, $p<0.0005$; Fig. $7 A$; and $F_{(4,32)}=123.8, p<0.0005$; Fig. $7 B)$. This increased sociality is consistent with previous work showing that play behavior increases during the juvenile period in mice (Terranova et al., 1993). The similarities in social behavior between normal PND 35 and adult mice in which neurogenesis was ablated during the juvenile period (compare PND 75 TK-GCV and PND 35 in Fig. 7 $A, B$ ) suggested that juvenile neurogenesis is required to support the normal increase in socialization seen during the juvenile period. The notion that neurons generated at around PND 30 are capable of integrating into circuits that modify behavior at PND 55 is consistent with studies showing that NSCs form significant synaptic connections after $21 \mathrm{~d}$ in adult animals, and that this process is significantly faster in younger animals (OverstreetWadiche et al., 2006; Zhao et al., 2006; Whitman and Greer, 2007). Maturation of social behavior was not associated with alteration in the latency to find food $\left(F_{(4,32)}=0.229, p=0.92\right.$; Fig. $7 C)$, consistent with previous studies showing that the MOB is fully functional at PND 35 (Yamazaki et al., 2000; Sullivan and Holman, 2010). Finally, there were no differences between the groups in the time spent investigating an unfamiliar inanimate object, suggesting that this increase in sociality is 
A
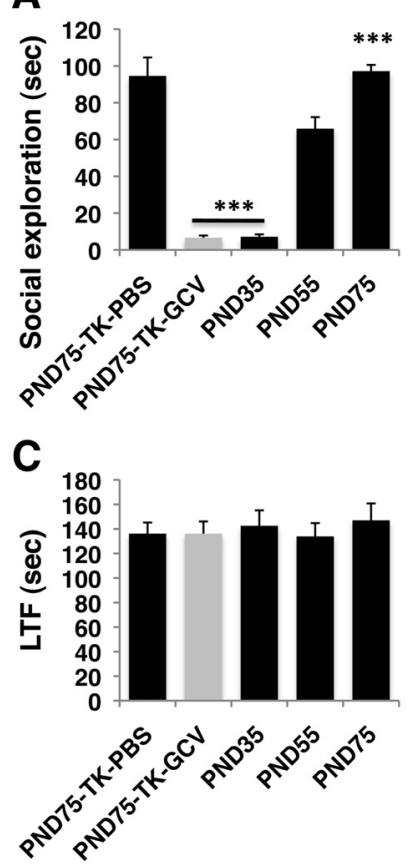

B
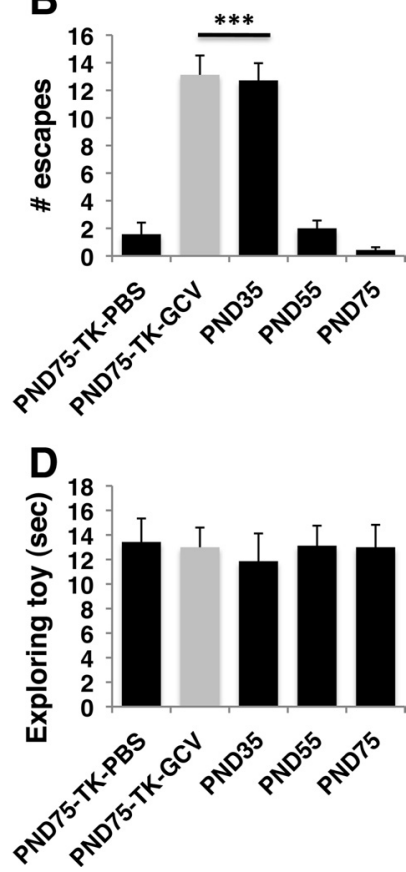

Figure 7. Juvenile neurogenesis is required to support the increase in socialization seen during this period. TK-GFP females were exposed to either intracerebroventricular PBS or GCV ( $n=8-7$ for each condition), singly housed, and tested in affiliative behavior on PND 75 (PND75-TK-PBS or PND75-TK-GCV). BALB/CByj wild-type females were singly housed on PND 27 and tested in the affiliative assay on either PND $35(n=7), \operatorname{PND} 55(n=8)$, or PND $75(n=7)$. Note that these mice were not exposed to surgery. $\boldsymbol{A}$, Social exploration increased with age. $\boldsymbol{B}$, Number of escapes decreased with age. Note that juvenile neurogenesis was intact in all groups except for the PND75-TK-GCV group (gray) and that social behavior of PND75-TK-GCV resembles that of PND 35 mice. $C$, Latency to find food (LTF). D, Time spent exploring inanimate object. ${ }^{* * *} p<0.0005$ compared with PND 55 group mice using Tukey-HSD post hoc analysis following one-way ANOVA.

not due to increased exploration of a novel object $\left(F_{(4,32)}=\right.$ 0.101, $p=0.98$; Fig. 7D).

\section{Discussion}

Here we show that neurogenesis during the juvenile period (i.e., PND 30-55) in female mice is essential for normal social development. Ablating neurogenesis in adulthood had no effect on social behaviors tested, suggesting that once matured, the expression of certain forms of social behavior no longer requires adult neurogenesis. These findings make three important contributions to previous work in this area. First, these studies suggest that juvenile neurogenesis is a cellular mechanism essential for the increase in socialization normally seen during this period in mammals. Second, while the functions of neurogenesis during embryogenesis, the perinatal period, and adulthood have been explored previously, juvenile neurogenesis has been largely ignored. Our work demonstrates the importance of this developmental process in programming complex behaviors in adulthood. Third, despite the well accepted notion that neurogenesis during different developmental phases makes distinct contributions to adult behavior, we are not aware of any previous work that directly test this hypothesis. Our finding that ablating neurogenesis during the juvenile period and adulthood causes divergent outcomes in some, but not all behaviors, demonstrates the complexity involved in answering this question.

Several lines of evidence suggest that the integration of new neurons into the MOB during the juvenile period plays an im-

portant role in the development of social behavior in female mice. First, activation of the MOB plays an important role in guiding diverse social behaviors in rodents (Baum and Kelliher, 2009). Second, rate of juvenile neurogenesis in the MOB is highly predictive of social behavior in adulthood (Fig. 5A). Third, a substantial number of the neurons generated in the MOB during the juvenile period are activated by a social encounter in adulthood (Fig. 6C), indicating that these newly formed neurons integrate into functional circuits that process social cues. Fourth, levels of c-fos activation in the MOB were similarly low in females with juvenile ablation of neurogenesis exposed to an unfamiliar female in their home cage and females that were kept socially isolated (Fig. 6), consistent with a defect in detecting olfactory cues present in the cage. However, we currently cannot rule out the possibility that integration of nestin-positive NSC outside the MOB are required for the formation of circuits that promote social proximity and active social investigation, behaviors that might be necessary to increase c-fos in the MOB above background levels (see more on this issue below). Ablating juvenile neurogenesis does not cause anosmia, and normal PND 35 mice possess an intact sense of smell (Yamazaki et al., 2000; Sullivan and Holman, 2010), suggesting that if the social aversion seen in these mice is due to a defect in olfaction, it is likely to be a more subtle and specific olfactory function that matures during the juvenile period.

It is currently unclear whether ablating juvenile neurogenesis in the MOB is sufficient to induce the social abnormalities seen after exposure to GCV during the juvenile period. This is because GCV-induced blockade of nestin-positive NSC proliferation also eliminates neurogenesis in the hippocampus and decreases NSC survival in the corpus callosum (Fig. 2), processes that may also contribute to the observed social deficits. In fact, there are good reasons to suspect that juvenile neurogenesis in the hippocampus also plays an important role in the development of normal social behavior in adulthood. For example, levels of juvenile neurogenesis in the hippocampus are highly correlated with levels of social exploration and escape behavior in adulthood (Fig. 5B), and social encounter induces robust c-fos activation in the hippocampus in a manner that required juvenile neurogenesis (Fig. $6 F$ ). In addition, the behavioral consequences of ablating juvenile neurogenesis are reminiscent of those described for neonatal lesions to the ventral hippocampus (Lipska and Weinberger, 2000). For example, both manipulations are associated with an increase in exploratory behavior and a defect in social behavior in adulthood (Sams-Dodd et al., 1997; Becker et al., 1999; Silva-Gómez et al., 2003; Flores et al., 2005). Importantly, lesions to the ventral hippocampus in neonates, but not adult mice, decrease social exploration in adulthood (Becker et al., 1999), consistent with the notion that the developing hippocampus plays an important role in the capacity to process social information (Maaswinkel et al., 1997). It is therefore possible that juvenile neurogenesis in the ventral hippocampus is necessary to promote active social investigation of other adult females, a behavior that in turn is necessary to induce c-fos activation in the MOB. Interestingly, the ventral hippocampus makes direct connections with the olfactory bulb and other primary olfactory cortical areas (Fanselow and Dong, 2010), raising the possibility that juvenile neurogenesis in the $\mathrm{MOB}$ and the hippocampus function conjointly to program adult social behavior. Additional work using localized ablation of juvenile neurogenesis in the hippocampus, $\mathrm{MOB}$, and both sites is needed to clarify the exact contribution that each neurogenic region makes to adult social competency.

Blocking neurogenesis in the juvenile and adult was associated with similar outcomes in the open-field assay, dark-light test, and 
object-recognition tasks. These findings are consistent with previous results showing that eliminating neurogenesis impairs hippocampal-dependent function (Shors et al., 2001; Saxe et al., 2006; Imayoshi et al., 2008; Clelland et al., 2009), but has no effect on exploratory behavior in the open-field test (Saxe et al., 2006). However, this is the first report showing that eliminating adult neurogenesis increases exploratory behavior in the dark-light test and is inconsistent with previous work (Saxe et al., 2006; Schloesser et al., 2010). The replication of these results in two cohorts of juvenile mice and in one cohort of adult mice demonstrate that at least in females from this strain, the findings are robust and reproducible. Moreover, TK-GFP and wild-type mice not exposed to intracerebroventricular GCV showed no differences in this task, indicating that the presence of the transgene is not sufficient to induce these behavioral changes (Table 1). We believe that these different outcomes might be due to the gender, strain of mice, or experimental conditions used here (e.g., lighting conditions, relative sizes of the dark-light chambers, and length of testing).

The finding that juvenile neurogenesis make critical contribution to social competency in adulthood may provide novel insights into human psychopathologies that first emerge during the peripubescent period. First, the motivation and the ability to socialize with conspecifics is essential for reproduction, rearing offspring, and forming stable social networks in all mammals, suggesting that some aspects of social development may be conserved among rodents and humans (Insel and Fernald, 2004). Indeed, increased play behavior is conspicuous during the juvenile period in all mammals, consistent with the notion that this developmental phase provides an important window for socialization (Meaney et al., 1985; Bekoff and Collin, 1998). Second, similar patterns of neurogenesis are present during the postnatal period in rodents, nonhuman primates, and humans (Pencea et al., 2001; Curtis et al., 2007). These include the restriction of neurogenesis to the SGZ and SVZ and the dramatic decline in levels of neurogenesis between the juvenile period and adulthood. Third, levels of neurogenesis are lower in individuals with schizophrenia (Reif et al., 2006) and the symptoms of this illness first emerge during adolescence, raising the possibility that abnormal levels of neurogenesis during this developmental stage may contribute to the cognitive and social deficits seen in this illness. Studies that characterize the function of early postnatal neurogenesis in guiding normal social development in nonhuman primates and humans may reveal novel cellular mechanisms to explain the abnormal social development seen in some forms of schizophrenia and autism spectrum disorder. These observations underscore the importance of neurodevelopmental changes occurring during the juvenile period, with present findings highlighting neurogenesis as an important cellular mechanism that mediates the maturation of adult-like social behavior in female mice.

\section{References}

Altman J, Das GD (1965) Post-natal origin of microneurones in the rat brain. Nature 207:953-956.

Barthelemy M, Gourbal BE, Gabrion C, Petit G (2004) Influence of the female sexual cycle on BALB/c mouse calling behaviour during mating. Naturwissenschaften 91:135-138.

Batista-Brito R, Close J, Machold R, Fishell G (2008) The distinct temporal origins of olfactory bulb interneuron subtypes. J Neurosci 28:3966-3975.

Baum MJ, Kelliher KR (2009) Complementary roles of the main and accessory olfactory systems in mammalian mate recognition. Annu Rev Physiol 71:141-160.

Becker A, Grecksch G, Bernstein HG, Höllt V, Bogerts B (1999) Social be- haviour in rats lesioned with ibotenic acid in the hippocampus: quantitative and qualitative analysis. Psychopharmacology (Berl) 144:333-338.

Bekoff M, Collin A (1998) Intentional communication and social play: how and why animals negotiate and agree to play. Cambridge: Cambridge UP.

Breton-Provencher V, Lemasson M, Peralta MR 3rd, Saghatelyan A (2009) Interneurons produced in adulthood are required for the normal functioning of the olfactory bulb network and for the execution of selected olfactory behaviors. J Neurosci 29:15245-15257.

Brewster ME, Raghavan K, Pop E, Bodor N (1994) Enhanced delivery of ganciclovir to the brain through the use of redox targeting. Antimicrob Agents Chemother 38:817-823.

Bush TG, Savidge TC, Freeman TC, Cox HJ, Campbell EA, Mucke L, Johnson MH, Sofroniew MV (1998) Fulminant jejuno-ileitis following ablation of enteric glia in adult transgenic mice. Cell 93:189-201.

Caligioni CS (2009) Assessing reproductive status/stages in mice. Curr Protoc Neurosci Appendix 4:Appendix 4I.

Cerghet M, Skoff RP, Bessert D, Zhang Z, Mullins C, Ghandour MS (2006) Proliferation and death of oligodendrocytes and myelin proteins are differentially regulated in male and female rodents. J Neurosci 26:1439-1447.

Clelland CD, Choi M, Romberg C, Clemenson GD Jr, Fragniere A, Tyers P, Jessberger S, Saksida LM, Barker RA, Gage FH, Bussey TJ (2009) A functional role for adult hippocampal neurogenesis in spatial pattern separation. Science 325:210-213.

Curtis MA, Eriksson PS, Faull RL (2007) Progenitor cells and adult neurogenesis in neurodegenerative diseases and injuries of the basal ganglia. Clin Exp Pharmacol Physiol 34:528-532.

Doetsch F, Hen R (2005) Young and excitable: the function of new neurons in the adult mammalian brain. Curr Opin Neurobiol 15:121-128.

Drickamer LC (1974) Contact stimulation, androgenized females and accelerated sexual maturation in female mice. Behav Biol 12:101-110.

Fanselow MS, Dong HW (2010) Are the dorsal and ventral hippocampus functionally distinct structures? Neuron 65:7-19.

Flores G, Silva-Gómez AB, Ibáñez O, Quirion R, Srivastava LK (2005) Comparative behavioral changes in postpubertal rats after neonatal excitotoxic lesions of the ventral hippocampus and the prefrontal cortex. Synapse 56:147-153.

Fowler CD, Liu Y, Ouimet C, Wang Z (2002) The effects of social environment on adult neurogenesis in the female prairie vole. J Neurobiol $51: 115-128$

Hatten ME (1999) Central nervous system neuronal migration. Annu Rev Neurosci 22:511-539.

Henderson ND, Turri MG, DeFries JC, Flint J (2004) QTL analysis of multiple behavioral measures of anxiety in mice. Behav Genet 34:267-293.

Imayoshi I, Sakamoto M, Ohtsuka T, Takao K, Miyakawa T, Yamaguchi M, Mori K, Ikeda T, Itohara S, Kageyama R (2008) Roles of continuous neurogenesis in the structural and functional integrity of the adult forebrain. Nat Neurosci 11:1153-1161.

Insel TR, Fernald RD (2004) How the brain processes social information: searching for the social brain. Annu Rev Neurosci 27:697-722.

Lazarini F, Mouthon MA, Gheusi G, de Chaumont F, Olivo-Marin JC, Lamarque S, Abrous DN, Boussin FD, Lledo PM (2009) Cellular and behavioral effects of cranial irradiation of the subventricular zone in adult mice. PLoS One 4:e7017.

Lemasson M, Saghatelyan A, Olivo-Marin JC, Lledo PM (2005) Neonatal and adult neurogenesis provide two distinct populations of newborn neurons to the mouse olfactory bulb. J Neurosci 25:6816-6825

Li Y, Mu Y, Gage FH (2009) Development of neural circuits in the adult hippocampus. Curr Top Dev Biol 87:149-174.

Lipska BK, Weinberger DR (2000) To model a psychiatric disorder in animals: schizophrenia as a reality test. Neuropsychopharmacology 23:223-239.

Maaswinkel H, Gispen WH, Spruijt BM (1997) Executive function of the hippocampus in social behavior in the rat. Behav Neurosci 111:777-784.

Magavi SS, Mitchell BD, Szentirmai O, Carter BS, Macklis JD (2005) Adultborn and preexisting olfactory granule neurons undergo distinct experiencedependent modifications of their olfactory responses in vivo. J Neurosci 25:10729-10739.

Maggio JC, Whitney G (1985) Ultrasonic vocalizing by adult female mice (Mus musculus). J Comp Psychol 99:420-436.

Mak GK, Enwere EK, Gregg C, Pakarainen T, Poutanen M, Huhtaniemi I, Weiss S (2007) Male pheromone-stimulated neurogenesis in the adult 
female brain: possible role in mating behavior. Nat Neurosci 10:1003-1011.

Mathis C, Hindelang C, LeMeur M, Borrelli E (2000) A transgenic mouse model for inducible and reversible dysmyelination. J Neurosci 20:7698-7705.

Meaney MJ, Stewart J, WWB (1985) Sex differnces in social play: the socialization of sex roles. In: Advances in the study of behavior (Rosenblatt J, Beer C, Busnel MC, Slater PJ, eds), pp 1-58: New York: Academic.

Overstreet-Wadiche LS, Bensen AL, Westbrook GL (2006) Delayed development of adult-generated granule cells in dentate gyrus. J Neurosci $26: 2326-2334$.

Pencea V, Bingaman KD, Freedman LJ, Luskin MB (2001) Neurogenesis in the subventricular zone and rostral migratory stream of the neonatal and adult primate forebrain. Exp Neurol 172:1-16.

Pfaffl MW (2001) A new mathematical model for relative quantification in real-time RT-PCR. Nucleic Acids Res 29:e45.

Reif A, Fritzen S, Finger M, Strobel A, Lauer M, Schmitt A, Lesch KP (2006) Neural stem cell proliferation is decreased in schizophrenia, but not in depression. Mol Psychiatry 11:514-522.

Sams-Dodd F, Lipska BK, Weinberger DR (1997) Neonatal lesions of the rat ventral hippocampus result in hyperlocomotion and deficits in social behaviour in adulthood. Psychopharmacology (Berl) 132:303-310.

Saxe MD, Battaglia F, Wang JW, Malleret G, David DJ, Monckton JE, Garcia AD, Sofroniew MV, Kandel ER, Santarelli L, Hen R, Drew MR (2006) Ablation of hippocampal neurogenesis impairs contextual fear conditioning and synaptic plasticity in the dentate gyrus. Proc Natl Acad Sci U S A 103:17501-17506.

Schloesser RJ, Lehmann M, Martinowich K, Manji HK, Herkenham M (2010) Environmental enrichment requires adult neurogenesis to facilitate the recovery from psychosocial stress. Mol Psychiatry 15:1152-1163.

Shingo T, Gregg C, Enwere E, Fujikawa H, Hassam R, Geary C, Cross JC, Weiss S (2003) Pregnancy-stimulated neurogenesis in the adult female forebrain mediated by prolactin. Science 299:117-120.

Shors TJ, Miesegaes G, Beylin A, Zhao M, Rydel T, Gould E (2001) Neurogenesis in the adult is involved in the formation of trace memories. Nature 410:372-376.
Silva-Gómez AB, Bermudez M, Quirion R, Srivastava LK, Picazo O, Flores G (2003) Comparative behavioral changes between male and female postpubertal rats following neonatal excitotoxic lesions of the ventral hippocampus. Brain Res 973:285-292.

Singer BH, Jutkiewicz EM, Fuller CL, Lichtenwalner RJ, Zhang H, Velander AJ, Li X, Gnegy ME, Burant CF, Parent JM (2009) Conditional ablation and recovery of forebrain neurogenesis in the mouse. J Comp Neurol 514:567-582.

Sullivan RM, Holman PJ (2010) Transitions in sensitive period attachment learning in infancy: the role of corticosterone. Neurosci Biobehav Rev 34:835-844.

Terranova ML, Laviola G, Alleva E (1993) Ontogeny of amicable social behavior in the mouse: gender differences and ongoing isolation outcomes. Dev Psychobiol 26:467-481.

Thornton JE, Finn PD (1999) Estrus. In: Encyclopedia of reproduction (Knobil E, Neill JD, eds), pp 136-141. Oxford: Academic.

Valley MT, Mullen TR, Schultz LC, Sagdullaev BT, Firestein F (2009) Ablation of mouse adult neurogenesis alters olfactory bulb structure and olfactory fear conditioning. Front Neurosci 3:51.

Wei L, David A, Duman RS, Anisman H, Kaffman A (2010) Early life stress increases anxiety-like behavior in BALBc mice despite a compensatory increase in levels of postnatal maternal care. Horm Behav 57:396-404.

Whitman MC, Greer CA (2007) Synaptic integration of adult-generated olfactory bulb granule cells: basal axodendritic centrifugal input precedes apical dendrodendritic local circuits. J Neurosci 27:9951-9961.

Yamazaki K, Beauchamp GK, Curran M, Bard J, Boyse EA (2000) Parentprogeny recognition as a function of MHC odortype identity. Proc Natl Acad Sci U S A 97:10500-10502.

Zhao C, Teng EM, Summers RG Jr, Ming GL, Gage FH (2006) Distinct morphological stages of dentate granule neuron maturation in the adult mouse hippocampus. J Neurosci 26:3-11.

Zimmerman L, Parr B, Lendahl U, Cunningham M, McKay R, Gavin B, Mann J, Vassileva G, McMahon A (1994) Independent regulatory elements in the nestin gene direct transgene expression to neural stem cells or muscle precursors. Neuron 12:11-24. 